\title{
Antineoplastic drugs contamination of workplace surfaces in two Portuguese hospitals
}

\section{Susana Viegas, Mário Pádua, Ana Costa Veiga, Elisabete Carolino \& Mário Gomes}

\section{Environmental Monitoring and} Assessment

An International Journal Devoted to Progress in the Use of Monitoring Data in Assessing Environmental Risks to Man and the Environment

ISSN 0167-6369

Volume 186

Number 11

Environ Monit Assess (2014)

186:7807-7818

DOI 10.1007/s10661-014-3969-1

\section{ENVIRONMENTAL MONITORING AND ASSESSMENT}

\begin{tabular}{lr}
\hline An International Journal devoted to progress in the use of monitoring \\
data in assessing environmental risks to Man and the environment.
\end{tabular}$\quad \begin{array}{r}\text { ISSN 0167-6369 } \\
\text { CODEN EMASDH }\end{array}$

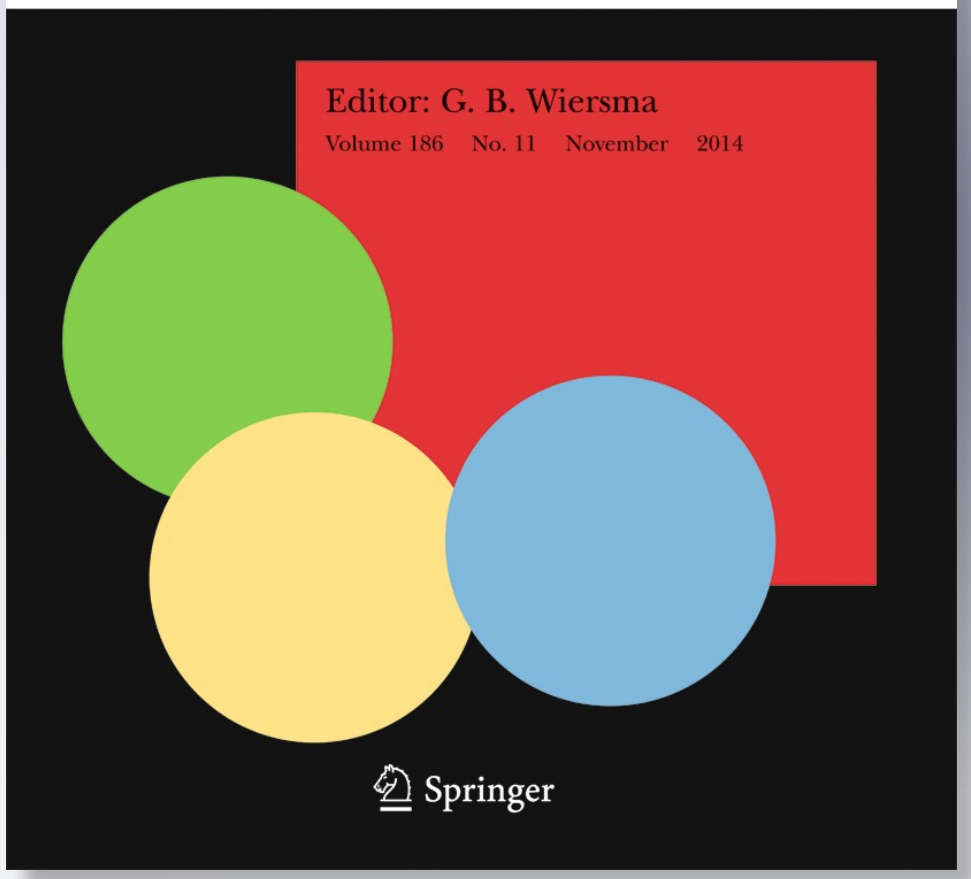

\section{自 Springer}


Your article is protected by copyright and all rights are held exclusively by Springer International Publishing Switzerland. This eoffprint is for personal use only and shall not be self-archived in electronic repositories. If you wish to self-archive your article, please use the accepted manuscript version for posting on your own website. You may further deposit the accepted manuscript version in any repository, provided it is only made publicly available 12 months after official publication or later and provided acknowledgement is given to the original source of publication and a link is inserted to the published article on Springer's website. The link must be accompanied by the following text: "The final publication is available at link.springer.com". 


\title{
Antineoplastic drugs contamination of workplace surfaces in two Portuguese hospitals
}

\author{
Susana Viegas • Mário Pádua • Ana Costa Veiga • \\ Elisabete Carolino • Mário Gomes
}

Received: 13 March 2014 / Accepted: 24 July 2014 / Published online: 6 August 2014

(C) Springer International Publishing Switzerland 2014

\begin{abstract}
Despite the classification as known or suspected human carcinogens, by the International Agency for Research on Cancer, the antineoplastic drugs are extensively used in cancer treatment due to their specificity and efficacy. As human carcinogens, these drugs represent a serious threat to the healthcare workers involved in their preparation and administration. This work aims to contribute to better characterize the occupational exposure of healthcare professionals to antineoplastic drugs, by assessing workplace surfaces contamination of pharmacy and administration units of two Portuguese hospitals. Surface contamination was assessed by the determination of cyclophosphamide, 5fluorouracil, and paclitaxel. These three drugs were used as surrogate markers for surfaces contamination by cytotoxic drugs. Wipe samples were taken and analyzed by HPLCDAD. From the total of 327 analyzed samples, in 121 (37\%) was possible to detect and quantify at least one drug. Additionally, 28 samples (8.6\%) indicate contamination by more than one antineoplastic drug, mainly in the administration unit, in both hospitals. Considering the findings in both hospitals, specific measures should be taken, particularly those related with the promotion of good practices and
\end{abstract}

S. Viegas · M. Pádua $\cdot$ E. Carolino $\cdot$ M. Gomes

RG Environment and Health. Lisbon School of Health

Technology. Polytechnic Institute of Lisbon, Lisbon, Portugal

\section{S. Viegas $(\bowtie)$}

Center for Malaria \& Tropical Diseases (CMDT) - Public

Health and Policy, Escola Nacional de Saúde Pública,

Universidade Nova de Lisboa, Lisbon, Portugal

e-mail: susana.viegas@estesl.ipl.pt

A. C. Veiga

Lisbon School of Health Technology- Polytechnic Institute of Lisbon, Lisbon, Portugal safety procedures and also routine monitoring of surfaces contamination in order to guarantee the appliance of safety measures.

Keywords Cytotoxic drugs · Surfaces contamination . Wipe sampling · Occupational exposure assessment

\section{Introduction}

Antineoplastic drugs are essential for cancer treatment. Nevertheless, they can pose a serious threat to the healthcare workers involved in their preparation and administration (Shirato 1992; McDevitt et al. 1993; Connor and McDiarmid 2006; Yoshida et al. 2013), since most of them are classified by the International Agency for Research on Cancer as known or suspected human carcinogens (IARC 2012).

The risk associated with occupational exposure mostly arises from chronic exposure to these agents. Over the last 30 years, particularly in Europe and North America, as awareness increased, numerous safety guidelines have been developed and published, aiming the protection of workers health, specifically diseases associated with these occupational settings. However, and despite the investment made in personal protective equipment, biological safety cabinets and workers training, occupational exposure of health care workers is still being reported (Fransman et al. 2007; Hedmer et al. 2008; Connor et al. 2010; Sugiura et al. 2011; Kopp et al. 2013; Miyake et al. 2013).

Recent studies have shown that workplace contamination due to poor work practices is a major cause of occupational exposure (Connor et al. 2010; Kiffmeyer 
et al. 2013). It may be impossible to eliminate all risk; however, it is imperious to reduce risk to the lowest level (Davis et al. 2011).

Exposure to these drugs can occur essentially by three ways: inhalation of aerosolized drugs; dermal absorption caused by direct skin contact; and, less frequently, ingestion due to contaminated hands (Turci et al. 2003). Presently, due to the evolution of the technical protection resources available in the workplaces, such as biological safety cabinets (BSC) and hoods, the most common route of exposure is skin absorption rather than inhalation or ingestion (Minoia et al. 1998). Recent studies showed also that antineoplastic levels in air samples were not significantly detected, suggesting that inhalation might have a lower impact when assessing exposure (Mason et al. 2005; Connor et al. 2010; Huang et al. 2012). Therefore, contact with contaminated workplace surfaces without gloves seems to play an important role in the dermal exposure to antineoplastic drugs (Hirst et al. 1984; Sessink et al. 1994; Kromhout et al. 2000; Fransman et al. 2005; Schierl et al. 2009). In this context, most of the recently published studies give particular attention to the assessment of surfaces contamination and report a generalized contamination, even in hospitals with the best equipment and strict safety procedures (Turci et al. 2003; Hedmer et al. 2005; Mason et al. 2005; Brouwers et al. 2007; Hon et al. 2011, 2013; Kopp et al. 2013). Moreover, since drugs are expected to be handled during both preparation and administration, it is important to differentiate pharmacy and patient wards (Hon et al. 2011; Kopp et al. 2013).

This work aims to contribute to better characterize the occupational exposure of healthcare professionals to antineoplastic drugs, by assessing workplace surfaces contamination of pharmacy and administration units of two Portuguese hospitals. Additionally, it is intended to discuss the factors that may be associated with surface contamination.

\section{Materials and methods}

Cyclophosphamide (CP), 5-fluorouracil (5FU), and paclitaxel (PTX) were used as surrogate markers for surfaces contamination by all cytotoxic drugs.

Selection of sampling surfaces

Before determining workplace contamination, it was necessary to understand where and how the workers can be in contact with contaminated surfaces in those units. Each hospital unit was visited at least in three separate occasions during the year of 2012. Direct and passive observations at each site were conducted by members of the research team to visually identify which surfaces/objects may be contaminated with antineoplastic drugs. A flowchart (not shown) was developed for each unit describing the antineoplastic drugs route from the pharmacy to the patient administration unit, and what surfaces/objects came into contact with the drug products and are touch/handled by the health staff.

The selected sampling spots (Tables 1 and 2) were judged to be the surfaces more potentially contaminated and, simultaneously, more frequently handled/touched by the health workers in the course of their routine tasks.

\section{Wipe sampling}

The surfaces were sampled in the first 3 months of the year 2013 in two different days in each hospital. The days were selected based on the workers classification as "normal" regarding preparation and administration of drugs.

Also, during the sampling period, data about which drugs were handled was collected.

Surface areas of $100 \mathrm{~cm}^{2}$, defined by a stainless frame with an internal size of $10 \times 10 \mathrm{~cm}$ were wipe-sampled with gauze moistened with ethyl-acetate as described elsewhere (Schmaus et al. 2002). The frame was cleaned between each sample, and a new pair of gloves was used for each wipe sample. All wipe samples were collected by the same person to guarantee reliable sampling. In the case of irregular surfaces such as phones, armrests, and handles, the wiped area was estimated and the result employed to calculate the contamination.

Table 1 Sampling sites considered relevant in hospital A $(n=67)$

\begin{tabular}{ll}
\hline Sampling sites & Unit \\
\hline Transfer (shelf and door handles) & Preparation \\
Cleanroom (worktops, trays, door handles) & Preparation \\
Anteroom (worktops, trays) & Preparation \\
Storage cabinet (shelf and door handles) & Preparation \\
Worktops (tables) & Administration \\
Administration devices (infusion pumps) & Administration \\
Transfer (shelf and door handles) & Administration \\
\hline
\end{tabular}


Table 2 Sampling sites considered relevant in hospital B $(n=260)$

\begin{tabular}{ll}
\hline Sampling sites & Unit \\
\hline $\begin{array}{l}\text { Transfer (shelf and door handles) } \\
\begin{array}{l}\text { Cleanroom (front barrier grille of the BSC, } \\
\text { chairs, worktops, trays, door handles) }\end{array}\end{array}$ & $\begin{array}{l}\text { Preparation } \\
\text { Anteroom (worktops) }\end{array}$ \\
$\begin{array}{l}\text { Storage cabinet (shelf and door handles) } \\
\text { Checking room (phone, desks and worktops, chairs, } \\
\quad \text { calculators and PC) }\end{array}$ & Preparation \\
$\begin{array}{l}\text { Worktops (tables and cars) } \\
\text { Administration devices (control panels, } \\
\quad \text { infusion pumps) }\end{array}$ & Preparation \\
$\begin{array}{l}\text { Treatment chairs (armrests) and beds } \\
\text { Personal protection equipment (gloves) } \\
\text { Drug container (packing after preparation } \\
\text { and box for manual transportation) }\end{array}$ & Administration \\
\hline
\end{tabular}

Wipe sample extraction

All wipe samples were extracted as described elsewhere (Schmaus et al. 2002). The wipes were extracted with $15 \mathrm{~mL}$ of acetonitrile/methanol/water (10:25:65) for $20 \mathrm{~min}$ at room temperature in a bottleroller homogenizer. Extracts were filtered through a $0.22-\mu \mathrm{m}$ filter prior to injection. Blank wipes were extracted according to the procedure described above and analyzed revealing no impurities that could interfere with the determination of the antineoplastic drugs (different retention times in the chromatogram).

\section{HPLC-DAD separation and quantification}

Separation and quantification was performed according to Larson and colleagues (2002) on a ThermoUNICAM Surveyor HPLC-DAD; $100-\mu \mathrm{L}$ sample loop; column Hypersil-GOLD $15 \times 5 \times 4.6$ with a guard column; mobile phase of acetonitrile/methanol/water (19:13:68) at a flow of $0.8 \mathrm{~mL} \mathrm{~min}^{-1}$. All HPLC grade solvents were purchased from VWR. CP, 5FU, and PTX were purchased from Sigma. For all the drugs, calibration curves were performed after extraction of spiked wipes. Each sample was injected in triplicate. Results are the mean with RSD under $5 \%$. LOD and LOQ were defined for each drug (Table 3). Concentrations above LOQ were considered quantified. The values below LOQ were considered detected but not quantifiable and are reported as $<\mathrm{LOQ}$. Values below LOD were considered non-detected and are reported as ND.
Statistical analysis

Data analysis was performed using SPSS version 22.0. For comparison of two independent groups, we used the Mann-Whitney $U$ test. For comparison of the fraction of surfaces where contamination had values below LOQ and a comparison of the fraction of surfaces where contamination had values above LOQ, between preparation and administration units in each hospital, we used the equality of proportions test with two independent samples.

Ethical considerations

This study was approved by the respective Institutional Ethic Boards of both hospitals and by Portuguese Authority for Work Conditions.

\section{Results}

Setting

The two hospitals enrolled on the study present very similar working practices: the preparation is centralized in one unit controlled by the pharmacist and prepared by pharmacy technicians; the administration of cytotoxic drugs is the responsibility of nursing staff.

Main characteristics of both hospitals

In hospital A, between the checking room and the cleanroom exists a transfer system/pass through hatches, which allows a separation of drugs for preparation and admixtures for administration. The preparation unit is linked to the oncology ward, where the administration takes place, also through a transfer system. The transport of cytotoxics for the oncology ward does not occur before the preparation is finished. After preparation, the nurses collect it from the transfer in trays.

In hospital B, the preparation unit is centralized in the pharmacy, and the admixtures are carried in hard-walled and robust containers to the administration unit.

Both hospitals use class II BSC in a grade B room for the preparation of cytotoxic drugs, two in hospital A, and three in hospital B. In the preparation unit, personal protective equipment for pharmacy technicians includes overshoes, head covering, goggles, mask P2/P3, sterile gown, and sterile gloves (two pairs). The pharmacists, in 
Table 3 Analytical data

$L O D$ detection limit, $L O Q$ quantification limit

\begin{tabular}{llll}
\hline Drug & $\mathrm{CP}$ & $5 \mathrm{FU}$ & PTX \\
\hline LOD & $0.10 \mu \mathrm{g} / \mathrm{cm}^{2}$ & $3.30 \mathrm{ng} / \mathrm{cm}^{2}$ & $0.167 \mathrm{ng} / \mathrm{cm}^{2}$ \\
LOQ & $0.30 \mu \mathrm{g} / \mathrm{cm}^{2}$ & $10.00 \mathrm{ng} / \mathrm{cm}^{2}$ & $0.50 \mathrm{ng} / \mathrm{cm}^{2}$ \\
$R^{2}$ & 0.9937 & 0.9933 & 0.9978 \\
Peak area & $4,236.43+639,570 \times \mathrm{CP}$ & $-129,764+127,590 \times 5 \mathrm{FU}$ & $2,903.38+219,663 \times \mathrm{PTX}$ \\
\hline
\end{tabular}

the checking room, wear one pair of gloves as the nursing staff in administration ward.

Top five drugs prepared in both hospitals include $5 F U, C P$, and PTX. The mean number of drug preparations/day is approximately 60 , with a team of five pharmacy technicians, two pharmacists and five nurses in an 8-h shift.

Cleaning procedures are also similar for both hospitals. Pharmacy technicians are responsible for cleaning and disinfection of BSC, but the facilities of the preparation and administration units are cleaned by an outsourced cleaning service.

From the 327 analysed samples of both hospitals, $121(37 \%)$ were positive (>LOQ). A sample was regarded as positive, when at least one of the three surrogate markers was detected and quantified.

Besides this and although impossible to quantify, there were more 117 samples $(35.8 \%)$ that showed also the presence $(<\mathrm{LOQ})$ of one or more markers.

Considering hospital A, from the 67 samples, 21 $(31.3 \%)$ were positive and 13 of them $(19.4 \%)$ presented contamination with more than one drug. In the latter, $9(69.2 \%)$ were sampled in the administration unit. In hospital B, 100 (38.5\%) out of 260 samples were positive and $15(5.8 \%)$ showed contamination from more than one drug and $13(86.7 \%)$ of them were from the administration unit (Table 4).

Drug contamination results ( $>$ LOQ) were also compared between preparation and administration units in each hospital (Mann-Whitney $U$ test). In this case, 5FU $(U=276.0 ; p=0.001)$ and PTX $(U=344.5 ; p=0.003)$ contaminations were significantly different in both areas in hospital A. Regarding hospital B, PTX ( $U=4747.5$; $p=0.000)$ and $\mathrm{CP}(U=6706 ; p=0.025)$ also showed statistical differences between the two units.

In both hospitals, the administration unit showed higher values.

In addition and considering the results presented in Tables 5 and 6, we performed a comparison of the fraction of surfaces where contamination had values below LOQ and a comparison of the fraction of surfaces where contamination had values above LOQ, between preparation and administration units in each hospital (equality of proportions test with two independent samples). The results showed statistically significant differences between the preparation and the administration units in hospital B, verifying that the preparation has a significantly higher fraction of surfaces where contamination had values below LOQ $(z=3.191, p=0.001)$. Regarding fraction of surfaces where contamination had values above LOQ, statistically significant differences were found between the preparation and administration units on the 5FU $(z=-3.049, p=0.002)$ and on the Taxol $(z=-2.762,0=0.006)$ in the hospital A. In hospital B, also statistically significant differences were found on the 5FU $(z=-1.410, p=0.032)$ and on the Taxol $(z=-5.780, p<0.0001)$. In either case, the fraction of surfaces where contamination had values above LOQ is greater in the administration unit.

Surface contamination in hospital A

Considering hospital A (Table 5), in the samples of the administration areas, $3.7 \%$ samples contaminated with

Table 4 Results of surface contamination

\begin{tabular}{llllll}
\hline Hospitals & Samples with contamination & $\begin{array}{l}\text { Contamination with } \\
\text { more than 1 drug }\end{array}$ & CP & PFU & PTX \\
\hline A & $21 / 67(31.3 \%)$ & $13 / 67(19.4 \%)$ & $1 / 67(1.5 \%)$ & $17 / 67(25.4 \%)$ & $17 / 67(25.4 \%)$ \\
B & $100 / 260(38.5 \%)$ & $15 / 260(5.8 \%)$ & $14 / 260(5.4 \%)$ & $18 / 260(6.9 \%)$ & $54 / 260(27.3 \%)$ \\
Totals & $121 / 327(37 \%)$ & $28 / 327(8.6 \%)$ & $15 / 327(4.6 \%)$ & $35 / 327(10.7 \%)$ & $71 / 327(21.7 \%)$ \\
\hline
\end{tabular}


Table 5 Surface contamination level by each drug in Hospital A $(n=67)$

\begin{tabular}{|c|c|c|c|c|}
\hline \multirow[t]{2}{*}{ Unit } & \multirow[t]{2}{*}{ Surface } & \multicolumn{3}{|l|}{$\begin{array}{l}\text { Drug } \\
\mathrm{n} / \mathrm{n} \text { total }(\%) \text {-range }\end{array}$} \\
\hline & & $\mathrm{CP}$ & $5 \mathrm{FU}$ & PTX \\
\hline \multirow[t]{4}{*}{ Preparation $(n=40)$} & Transfer (shelf and door handles) & $\begin{array}{l}\text { Shelfs } \\
\text { ND 7/7 (100\%) } \\
\text { Door handles } \\
\text { ND 4/4 (100\%) }\end{array}$ & $\begin{array}{l}\text { Shelfs } \\
\text { ND 3/7 }(42.9 \%) \\
<\text { LOQ 3/7 }(42.9 \%) \\
>\text { LOQ } 1 / 7(14.3 \%) \\
13.7 \mathrm{ng} / \mathrm{cm}^{2} \\
\text { Door handles } \\
\quad \text { ND 4/4 }(100 \%)\end{array}$ & $\begin{array}{l}\text { Shelfs } \\
\text { ND 7/7 (100\%) } \\
\text { Door handles } \\
\text { ND 4/4 (100\%) }\end{array}$ \\
\hline & Cleanroom (worktops, trays, door handles) & $\begin{array}{l}\text { Worktops } \\
\text { ND 2/2 (100 \%) } \\
\text { Trays } \\
\text { ND 7/7 (100 \%) } \\
\text { Door handles } \\
\text { ND 2/2 (100\%) }\end{array}$ & $\begin{array}{l}\text { Worktops } \\
\text { ND 2/2 (100\%) } \\
\text { Trays } \\
\text { ND 7/7 (100\%) } \\
\text { Door handles } \\
\text { ND } 2 / 2(100 \%)\end{array}$ & $\begin{array}{l}\text { Worktops } \\
\text { ND 2/2 (100\%) } \\
\text { Trays } \\
\text { ND 7/7 (100\%) } \\
\text { Door handles } \\
\text { ND 2/2 (100\%) }\end{array}$ \\
\hline & Anteroom (worktops, trays) & $\begin{array}{l}\text { Worktops } \\
\text { ND 4/4 (100\%) } \\
\text { Trays } \\
\text { ND 2/2 (100\%) }\end{array}$ & $\begin{array}{l}\text { Worktops } \\
\text { ND 2/4 }(50 \%) \\
<\text { LOQ 2/4 }(50 \%) \\
\text { Trays } \\
\quad>\text { LOQ 2/2 }(100 \%) \\
14.4-15.8 \mathrm{ng} / \mathrm{cm}^{2}\end{array}$ & $\begin{array}{l}\text { Worktops } \\
\text { ND 4/4 }(100 \%) \\
\text { Trays } \\
\text { >LOQ 2/2 }(100 \%) \\
8.3-8.5 \mathrm{ng} / \mathrm{cm}^{2}\end{array}$ \\
\hline & Storage cabinet (shelf and door handles) & $\begin{array}{l}\text { Shelfs } \\
\text { ND 10/10 (100\%) } \\
\text { Door handles } \\
\text { ND } 2 / 2(100 \%)\end{array}$ & $\begin{array}{l}\text { Shelfs } \\
\text { ND 6/10 }(60 \%) \\
\text { <LOQ 2/10 (20\%) } \\
\text { >LOQ 2/10 (20\%) } \\
\text { 12.1-78.8 ng/cm² } \\
\text { Door handles } \\
\text { ND 2/2 }(100 \%)\end{array}$ & $\begin{array}{l}\text { Shelfs } \\
\text { ND 5/10 }(50 \%) \\
>\text { LOQ } 5 / 10(50 \%) \\
2.9-5.4 \mathrm{ng} / \mathrm{cm}^{2} \\
\text { Door handles } \\
\text { ND } 2 / 2(100 \%)\end{array}$ \\
\hline \multirow[t]{3}{*}{ Administration $(n=27)$} & Worktops (tables) & $\begin{array}{l}\text { ND 12/16 }(75 \%) \\
<\text { LOQ } 3 / 16(18.8 \%) \\
>\text { LOQ } 1 / 16(6.2 \%) \\
2.6 \mu \mathrm{g} / \mathrm{cm}^{2}\end{array}$ & $\begin{array}{l}\text { ND 5/16 }(31.3 \%) \\
<\text { LOQ 5/16 (31.3\%) } \\
>\text { LOQ 6/16 (37.4\%) } \\
10.6-60.5 \mathrm{ng} / \mathrm{cm}^{2}\end{array}$ & $\begin{array}{l}\text { ND 2/16 (12.5\%) } \\
\text { >LOQ 14/16 (87.5\%) } \\
5.22-13.0 \mathrm{ng} / \mathrm{cm}^{2}\end{array}$ \\
\hline & Administration devices: infusion pumps & ND 2/2 (100\%) & ND 2/2(100\%) & $\begin{array}{l}>\text { LOQ } 2 / 2(100 \%) \\
5.4-5.8 \mathrm{ng} / \mathrm{cm}^{2}\end{array}$ \\
\hline & Transfer (shelf and door handles) & $\begin{array}{l}\text { Shelfs } \\
\text { ND } 7 / 7(100 \%) \\
\text { Door handles } \\
\text { ND 2/2 (100\%) }\end{array}$ & $\begin{array}{l}\text { Shelfs } \\
\quad<\text { LOQ } 1 / 7(14.3 \%) \\
\quad>\text { LOQ } 6 / 7(85.7 \%) \\
11.8-15.2 \mathrm{ng} / \mathrm{cm}^{2} \\
\text { Door handles } \\
\quad \text { ND } 1 / 2 \\
\quad \text { LOQ } 1 / 2(50 \%)\end{array}$ & $\begin{array}{l}\text { Shelfs } \\
\quad>\text { LOQ } 7 / 7(100 \%) \\
1.5-4.1 \mathrm{ng} / \mathrm{cm}^{2} \\
\text { Door handles } \\
\quad \text { ND } 2(100 \%)\end{array}$ \\
\hline
\end{tabular}

$\mathrm{CP}(>\mathrm{LOQ})$ and $11 \%$ was detected $\mathrm{CP}(<\mathrm{LOQ}) .8 .1$ and $44.4 \% 5$ FU-contaminated samples were found in preparation and administration areas. 5FU was detected in both preparation and administration areas (11.3 and $26.0 \%$, respectively). PTX-contaminated samples (11.3 and $85.2 \%)$ were found in preparation and administration areas. The highest measured value for CP $\left(2.6 \mu \mathrm{g} / \mathrm{cm}^{2}\right)$ was obtained in a worktop that gives support to the drug administration process.

In the case of $5 \mathrm{FU}$, the highest value $\left(78.8 \mathrm{ng} / \mathrm{cm}^{2}\right)$ was found in the refrigerator closet where drugs are stored located in the preparation unit. The surface that obtained the highest measured value of PTX (19.7 ng/ $\mathrm{cm}^{2}$ ) was the transfer shelf from the administration unit.

Surface contamination in hospital B

Considering hospital B (Table 6), a 4.1 and $7 \%$ CP (>LOQ)-contaminated samples were found in preparation and administration areas. Additionally, was detected CP in both preparation and administration (4.1 and $16.5 \%$, respectively). 5FU-contaminated samples (6.9 and $7 \%$ ) were found in preparation and administration areas. Moreover, 5FU was also detected in other samples from both preparation and administration areas 
Table 6 Surface contamination level by each drug in Hospital B $(n=260)$

\begin{tabular}{|c|c|c|c|c|}
\hline \multirow[t]{2}{*}{ Unit } & \multirow[t]{2}{*}{ Surfaces } & \multicolumn{3}{|l|}{$\begin{array}{l}\text { Drug } \\
\mathrm{n} / \mathrm{n} \text { total }(\%) \text {-range }\end{array}$} \\
\hline & & $\mathrm{CP}$ & $5 \mathrm{FU}$ & PTX \\
\hline \multirow[t]{5}{*}{$\begin{array}{r}\text { Preparation } \\
\quad(n=145)\end{array}$} & Transfer (shelf and door handles) & $\begin{array}{l}\text { Shelfs } \\
\text { ND 12/16 }(75 \%) \\
\text { <LOQ } 1 / 16(6.3 \%) \\
\text { >LOQ 3/16 (18.2\%) } \\
0.42-2.1 \mu \mathrm{g} / \mathrm{cm}^{2} \\
\text { Door handles } \\
\text { ND } 12 / 12(100 \%)\end{array}$ & $\begin{array}{l}\text { Shelfs } \\
\text { ND } 8 / 16(50 \%) \\
<\text { LOQ } 7 / 16(43.8 \%) \\
\text { >LOQ } 1 / 16(6.2 \%) \\
11.17 \mathrm{ng} / \mathrm{cm}^{2} \\
\text { Door handles } \\
\text { ND 4/12 }(33.3 \%) \\
<\text { LOQ } 8 / 12(66.7 \%)\end{array}$ & $\begin{array}{l}\text { Shelfs } \\
\text { ND 16/16 }(100 \%) \\
\text { Door Handles: } \\
\text { ND } 7 / 12(58.3 \%) \\
\text { >LOQ } 5 / 12(41.7 \%) \\
1.9-3.3 \mathrm{ng} / \mathrm{cm}^{2}\end{array}$ \\
\hline & $\begin{array}{c}\text { Cleanroom (front barrier grille of the BSC, } \\
\text { chairs, worktops, trays, door handles) }\end{array}$ & $\begin{array}{l}\text { BSC } \\
\text { ND 11/12 }(91.6 \%) \\
<\text { LOQ 1/12 }(8.4 \%) \\
\text { Chairs } \\
\text { ND } 7 / 7(100 \%) \\
\text { Worktops } \\
\text { ND 44/44 (100\%) } \\
\text { Trays } \\
\text { ND 5/5 }(100 \%) \\
\text { Door handles } \\
\text { ND 4/6 }(66.7 \%) \\
<\text { LOQ 2/6 }(33.3 \%)\end{array}$ & $\begin{array}{l}\text { BSC } \\
\text { ND 7/12 }(58.3 \%) \\
\text { <LOQ 4/12 }(33.3 \%) \\
\text { >LOQ } 1 / 12(8.4 \%) \\
14.6 \mathrm{ng} / \mathrm{cm}^{2} \\
\text { Chairs } \\
\text { ND } 7 / 7(100 \%) \\
\text { Worktops } \\
\text { ND 34/44 }(77.3 \%) \\
\text { <LOQ } 9 / 44(20.5 \%) \\
\text { >LOQ } 1 / 44(2.3 \%) \\
\text { 20.4 ng/cm } 2 \\
\text { Trays: } \\
\text { <LOQ } 5 / 5(100 \%) \\
\text { Door handles } \\
\text { ND 3/6 }(50 \%) \\
\text { <LOQ 3/6 }(50 \%)\end{array}$ & $\begin{array}{l}\text { BSC } \\
\text { ND 12/12 (100\%) } \\
\text { Chairs } \\
\text { ND 7/7 }(100 \%) \\
\text { Worktops } \\
\text { ND 44/44 (100\%) } \\
\text { Trays } \\
\text { ND 5/5 }(100 \%) \\
\text { Door handles } \\
\text { ND 4/6 }(66.7 \%) \\
\text { >LOQ } 2 / 6(33.3 \%) \\
\text { 8.96-12.4 ng/cm } 2\end{array}$ \\
\hline & Anteroom (worktops) & ND 10/10 (100\%) & $\begin{array}{l}\text { ND } 6 / 10(60 \%) \\
<\operatorname{LOQ} 4 / 10(40 \%)\end{array}$ & ND 10/10 (100\%) \\
\hline & Storage cabinet (shelf and door handles) & $\begin{array}{l}\text { Shelfs } \\
\text { ND 6/6 (100\%) } \\
\text { Door handles } \\
\text { ND 6/7(85.7\%) } \\
<\text { LOQ } 1 / 7(14.3 \%)\end{array}$ & $\begin{array}{l}\text { Shelfs } \\
\quad \text { ND 3/6 }(50 \%) \\
<\text { LOQ 3/6 }(50 \%) \\
\text { Door handles } \\
\text { ND 6/7 }(85.7 \%) \\
\quad<\text { LOQ } 1 / 7(14.3 \%)\end{array}$ & $\begin{array}{l}\text { Shelfs } \\
\text { ND 4/6 }(66.7 \%) \\
\text { >LOQ 2/6 }(33.3 \%) \\
10.4-13.6 \mathrm{ng} / \mathrm{cm}^{2} \\
\text { Door handles } \\
\text { ND } 7 / 7(100 \%)\end{array}$ \\
\hline & $\begin{array}{l}\text { Checking room (phone, desks and } \\
\text { worktops, chairs, calculators and PC) }\end{array}$ & $\begin{array}{l}\text { Phone } \\
\text { ND 3/4 }(75 \%) \\
>\text { LOQ } 1 / 4(25 \%) \\
21.3 \mu \mathrm{g} / \mathrm{cm}^{2} \\
\text { Desks } \\
\text { ND 5/6 }(83.3 \%) \\
\text { <LOQ } 1 / 6(16.7 \%) \\
\text { Chairs } \\
\text { ND 6/6 }(100 \%) \\
\text { Calculators and PC } \\
\text { ND 2/4 }(50 \%) \\
\text { >LOQ } 2 / 4(50 \%) \\
\text { 10.0-13.4 } \mu \mathrm{g} / \mathrm{cm}^{2}\end{array}$ & $\begin{array}{l}\text { Phone } \\
\text { ND 1/4 }(25 \%) \\
\text { >LOQ 3/4 }(75 \%) \\
\text { Desks } \\
\quad \text { ND } 1 / 6(16.7 \%) \\
\text { <LOQ 4/6 }(66.7 \%) \\
\text { >LOQ } 1 / 6(16.6 \%) \\
11.9 \mathrm{ng} / \mathrm{cm}^{2} \\
\text { Chairs } \\
<\text { LOQ } 5 / 6(83.3 \%) \\
>\text { LOQ } 1 / 6(16.7 \%) \\
14.4 \mathrm{ng} / \mathrm{cm}^{2} \\
\text { Calculators and PC } \\
\text { ND } 1 / 4(25 \%) \\
<\text { LOQ } 1 / 4(25 \%) \\
\text { >LOQ } 2 / 4(50 \%) \\
10.0-13.4 \mathrm{ng} / \mathrm{cm}^{2}\end{array}$ & $\begin{array}{l}\text { Phone } \\
\text { ND 4/4 (100\%) } \\
\text { Desks } \\
\text { ND 6/6 }(100 \%) \\
\text { Chairs } \\
\text { ND 2/6 }(33.3 \%) \\
\text { >LOQ 4/6 (66.7\%) } \\
\text { 2.1-6.6 ng/cm } 2 \\
\text { Calculators and PC } \\
\text { ND 4/4 (100\%) }\end{array}$ \\
\hline Administration $(n=115)$ & Worktops (tables and cars) & $\begin{array}{l}\text { Tables } \\
\text { ND 29/41 }(70.7 \%) \\
\text { < LOQ 9/41 }(22 \%) \\
\text { >LOQ 3/41 }(7.3 \%) \\
3.7-20.6 \mu \mathrm{g} / \mathrm{cm}^{2} \\
\text { Cars } \\
\text { ND 14/21 }(66.7 \%) \\
\text { <LOQ 4/21 }(19.0 \%) \\
>\text { LOQ 3/21 }(14.3 \%) \\
0.4-2.1 \mu \mathrm{g} / \mathrm{cm}^{2}\end{array}$ & $\begin{array}{l}\text { Tables } \\
\text { ND 34/41 }(83 \%) \\
\text { < LOQ 6/41 }(14.6 \%) \\
\text { >LOQ } 1 / 41(2.4 \%) \\
12.0 \mathrm{ng} / \mathrm{cm}^{2} \\
\text { Cars } \\
\text { ND } 11 / 21(52.4 \%) \\
\text { < LOQ 6/21 }(28.6 \%) \\
>\text { LOQ } 4 / 21(19.0 \%) \\
12.4-33.9 \mathrm{ng} / \mathrm{cm}^{2}\end{array}$ & $\begin{array}{l}\text { Tables } \\
\text { ND 18/41 (43.9\%) } \\
\text { >LOQ 23/41(56.1\%) } \\
\text { 1.3-260.9 ng/cm } \\
\text { Cars } \\
\quad \text { ND 6/21 }(28.6 \%) \\
\text { >LOQ } 15 / 21(71.4 \%) \\
0.7-479.7 \mathrm{ng} / \mathrm{cm}^{2}\end{array}$ \\
\hline
\end{tabular}


Table 6 (continued)

\begin{tabular}{|c|c|c|c|c|}
\hline \multirow[t]{2}{*}{ Unit } & \multirow[t]{2}{*}{ Surfaces } & \multicolumn{3}{|l|}{$\begin{array}{l}\text { Drug } \\
\mathrm{n} / \mathrm{n} \text { total }(\%) \text {-range }\end{array}$} \\
\hline & & $\mathrm{CP}$ & $5 \mathrm{FU}$ & PTX \\
\hline & $\begin{array}{l}\text { Administration devices: Control } \\
\text { panels and Infusion pumps }\end{array}$ & $\begin{array}{l}\text { ND 22/25 }(88 \%) \\
<\text { LOQ 3/25 (12\%) }\end{array}$ & $\begin{array}{l}\text { ND } 12 / 25(48 \%) \\
<\text { LOQ } 8 / 25(32 \%) \\
\text { LOQ } 5 / 25(20 \%) \\
10.7-41.3 \mathrm{ng} / \mathrm{cm}^{2}\end{array}$ & $\begin{array}{l}\text { ND } 16 / 25(64 \%) \\
\text { >LOQ } 9 / 25(36 \%) \\
1.2-1131.4 \mathrm{ng} / \mathrm{cm}^{2}\end{array}$ \\
\hline & Treatment chairs (armrests) and beds & $\begin{array}{l}\text { Treatment chairs } \\
\text { ND 6/9 }(66.7 \%) \\
<\text { LOQ } 1 / 9(11.1 \%) \\
>\text { LOQ } 2 / 9(22.2 \%) \\
0.3-1.1 \mu \mathrm{g} / \mathrm{cm}^{2} \\
\text { Beds } \\
\quad \text { ND 2/2 }(100 \%)\end{array}$ & $\begin{array}{l}\text { Treatment chairs } \\
\text { ND 9/9 (100\%) } \\
\text { Beds } \\
\text { ND 2/2 (100\%) }\end{array}$ & $\begin{array}{l}\text { Treatment chairs } \\
\text { ND 9/9 }(100 \%) \\
\text { Beds } \\
\text { ND 2/2 }(100 \%)\end{array}$ \\
\hline & Personal Protection Equipment (gloves) & $\begin{array}{l}\text { ND } 8 / 10(80 \%) \\
<\text { LOQ } 2 / 10(20 \%)\end{array}$ & $\begin{array}{l}\text { ND } 9 / 10(90 \%) \\
\text { > LOQ } 1 / 10(10 \%) \\
11.4 \mathrm{ng} / \mathrm{cm}^{2}\end{array}$ & $\begin{array}{l}\text { ND } 5 / 10(50 \%) \\
>\text { LOQ } 5 / 10(50 \%) \\
14.7-414.7 \mathrm{ng} / \mathrm{cm}^{2}\end{array}$ \\
\hline & $\begin{array}{l}\text { Drug container (packing after preparation } \\
\text { and box for manual transportation) }\end{array}$ & $\begin{array}{l}\text { Packing } \\
\text { ND 5/5(100\%) } \\
\text { Box } \\
\quad \text { ND 2/2(100\%) }\end{array}$ & $\begin{array}{l}\text { Packing } \\
\text { ND 3/5(60\%) } \\
<\text { LOQ } 1 / 5(20 \%) \\
>\text { LOQ } 1 / 5(20 \%) \\
13.7 \mathrm{ng} / \mathrm{cm}^{2} \\
\text { Box } \\
\quad \text { ND } 1 / 2(50 \%) \\
>\text { LOQ } 1 / 2(50 \%) \\
14.6 \mathrm{ng} / \mathrm{cm}^{2}\end{array}$ & $\begin{array}{l}\text { Packing } \\
\quad \text { - LOQ 5/5 }(100 \%) \\
34.8-46.7 \mathrm{ng} / \mathrm{cm}^{2} \\
\text { Box } \\
\quad \text { ND 1/2 }(50 \%) \\
\text { > LOQ 1/2 }(50 \%) \\
6.3 \mathrm{ng} / \mathrm{cm}^{2}\end{array}$ \\
\hline
\end{tabular}

(37.2 and $18.3 \%$, respectively). PTX-contaminated samples ( 9 and $50.4 \%$ ) were found in preparation and administration areas. The highest measured value for $\mathrm{CP}$ $\left(21.3 \mu \mathrm{g} / \mathrm{cm}^{2}\right)$ was obtained on the phone of the checking room that gives direct support to the cleanroom from the preparation unit. In the case of $5 \mathrm{FU}$, the highest value $\left(41.3 \mathrm{ng} / \mathrm{cm}^{2}\right)$ was found in one control panel infusion pump in the administration unit. The surface that obtained the highest measured value of PTX $\left(1,131.4 \mathrm{ng} / \mathrm{cm}^{2}\right)$ was also in a infusion pump.

\section{Discussion}

Antineoplastic drugs play an important role in cancer treatment, however not without side effects linked to their carcinogenic, mutagenic, and teratogenic properties. These side effects represent a potential health risk for workers handling these drugs during preparation or administration procedures. Moreover, carcinogenic, mutagenic, and teratogenic effects usually do not depend on a minimum dose but instead on a prolonged exposure. Therefore, it can be concluded that there is no safety threshold dose concerning exposure to these drugs. It would be appropriate to apply the ALARA principle: keep exposure/contamination levels as low as reasonably achievable (Hon et al. 2013).

Although several antineoplastic drugs are commonly employed in these hospital settings, to assess exposure, we used three antineoplastic drugs, CP, 5FU, and PTX, as surrogate markers for exposure. This can be regarded as a limitation as it assumes that similar levels of contamination can be present for the other agents. Those drugs are among the most frequently employed, and in high amounts, every day in both hospitals. Most of the studies published concerning this issue used the same approach (Larson et al. 2003; Mason et al. 2005; Castiglia et al. 2008; Hedmer et al. 2005, 2008; Touzin et al. 2009; Kopp et al. 2013). Additionally, these drugs are also recommended as markers for chemical contamination monitoring by ISOPP (2007). Additionally, since the same aseptic techniques are employed in the preparation of admixtures containing these or other drugs, similar results are to be expected.

The sampling strategy followed is another potential limitation of our study as contaminated surfaces could have been overlooked. However, since the observations were performed on different days and the drug flow was well described, we believe that it was possible to identify the potentially contaminated surfaces and the ones 
that represent higher risk for workers due to the fact that they are frequently handled. Furthermore, we studied only surfaces that may be touched/handled by the workers and avoided surfaces such as floors of the preparation and administration units, accordingly to other studies (Schmaus et al. 2002; Hedmer et al. 2008; Castiglia et al. 2008; Kopp et al. 2013; Hon et al. 2013).

This type of information is very useful because it allows the identification of the "weak points" in the working and cleaning procedures and where preventive actions can have the best result regarding minimizing workplace contamination. Regarding this aspect, Touzin et al. (2009) mentioned that environmental monitoring can have an important role for protecting workers from exposure to chemical agents because it can identify surfaces with high risk and to assess if the control measures, such as surfaces cleaning, had the expected results.

In both hospitals, the administration unit obtained higher values for the three surrogate markers with the exception of 5FU in hospital B. These results are corroborated by Hedmer and colleagues (2008) and Fransman and colleagues (2004) that concluded that dermal exposure is common among oncology nurses. Contrary to our findings, Hon et al. (2013) suggested the drug preparation stage as the most probable to be contaminated due to the concentrated drugs that are routinely handled and mixed. However, our results can be explained by the fact that preparation units in Portugal implement a higher number of safety procedures, since this is a much regulated area audited frequently by Portuguese Authority of Medicines and Health Products. Another concurring factor was referred by Touzin et al. (2009): different job description and less training from the workers can also contribute to high levels of contamination.

Our results showed contamination by drugs on days when those analytes were not used at all in preparation and administration. The same kind of results was found in a study developed by Castiglia et al. (2008). These results probably are resulting from defective cleaning procedures that can be related with the detergents used and/or the frequency of the cleaning procedures and, also, the surfaces that are object of the cleaning actions. Similar results led other authors to the same type of conclusions (Hedmer et al. 2008; Touzin et al. 2010; Turci et al. 2011; Hon et al. 2013).
Moreover, our results showed different results in the number of surfaces with contamination between the drugs considered. In detail, it was possible to observe less CP-contaminated surfaces $(4.6 \%)$ than $5 \mathrm{FU}$ and PTX (10.7 and 26.9\%, respectively) (Table 4). This can be explained by the lower sensitivity of the CP method, leading to the incapacity to measure values below $30 \mu \mathrm{g}$, than in the cases of $5 \mathrm{FU}(\mathrm{LOQ}=1,000 \mathrm{ng})$ and PTX (LOQ=50 ng). Additionally, CP is unstable at room temperature (Hedmer et al. 2004). In an opposite way, PTX presented higher number of contaminated surfaces $(21.7 \%)$ when comparing with the other two drugs studied (4.6\% for $\mathrm{CP}$ and $10.7 \%$ for $5 \mathrm{FU}$ ) (Table 4). This result can be due to two different factors that characterize PTX: low aqueous solubility and high chemical stability. These two factors combined can explain the results because probably this drug is more persistent in the surfaces, resisting more easily to environment conditions and cleaning procedures (Yang et al. 2007).

Regarding hospital A, surfaces contaminated with all surrogate markers were found in both preparation and administration areas, although contamination was significantly higher in the administration area. In the preparation area, we found contamination in the refrigerator closet handle where drugs are stored with the primary packaging. As demonstrated in other studies (Delporte et al. 1999; Nygren et al. 2002; Favier et al. 2003; Mason et al. 2003; Hedmer et al. 2005; Connor et al. 2005; Touzin et al. 2009; Turci et al. 2011), probably, the package is the main source of contamination. Moreover, the handling of these primary packaging is done some times without protection gloves by the pharmacists and, before that, by the workers in the hospital storage unit. This raises concern related with the potential of other hospital areas to be contaminated with antineoplastic drugs and, consequently, an increase in number of workers being potentially exposed (Hon et al. 2011).

The highest contamination of PTX in hospital was determined on the transfer. The transfer is commonly sampled in other studies (Schierl et al. 2009; Connor et al. 2010; Hon et al. 2013) due to the high handling frequency of this equipment by the workers that prepare and administer the cytotoxic drugs. Here, we have to consider that probably contaminated worker's gloves and metal trays are responsible for the transfer contamination. This demonstrates that contamination can spread throughout both services. Widespread 
contamination was also observed in other studies, despite the implementation of safety procedures for handling antineoplastic drugs (Schmaus et al. 2002; Castiglia et al. 2008; Connor et al. 2010). Furthermore, and considering that the metal trays are used to transport drugs after preparation, a study developed by Hon and colleagues (2011) showed that the trays are the most frequently contacted surface in the medication circuit. Curiously, and regarding the trays contamination, Kopp et al. (2013) demonstrated that there were significantly lower levels of surfaces contamination when preparations, after use, were transported on a tray instead of per hand (Kopp et al. 2013). Probably, in the present research, the most important factor that is influencing surfaces contamination is the inadequate frequency of glove changing by workers.

Considering hospital B, as in hospital A, contamination was found for all surrogate markers in both preparation and administration areas. Nevertheless, in the administration units, we observed higher contamination level.

In detail, it is important to mention that the highest surface contamination results were obtained in samples from hospital B. The result of the phone from the checking room is particularly problematic since in this place there is no direct handling of cytotoxic drugs as they were already prepared in the cleanroom. Moreover, the phone is frequently used without gloves since it is on a desk where normally the registration of the drug prepared takes place. In fact, it is used by all the personnel that work in the checking room. In this case, the inappropriate surfaces cleaning together with the incorrect working procedures surely contributed for the contamination found. The same kind of results were observed in a study developed in Italian hospitals (Castiglia et al. 2008) that concluded that the cause of contamination of this type of surfaces (phones, chairs, closets, and others not directly related with drug preparation) were the workers gloves that were not removed immediately after drug handling. As in our study, other studies (Minoia et al. 1998; Mason et al. 2005; Fransman et al. 2004, 2007) found contamination on the worker's gloves. For instance, the gloves that was possible to sample in the administration unit of hospital $\mathrm{B}$, showed contamination by $5 \mathrm{FU}\left(11.4 \mathrm{ng} / \mathrm{cm}^{2}\right)$ and, also, high amounts of PTX $\left(414.7 \mathrm{ng} / \mathrm{cm}^{2}\right)$.

The higher results for 5FU and PTX in hospital B were found on equipment from the administration unit, namely in administration devices such as infusion pumps.
Once again, these results may be due to spillage during the administration process as well as to inadequate cleaning protocols or incorrect working procedures (Sessink et al. 1992; Acampora et al. 2005; Touzin et al. 2009; Turci et al. 2011; Hon et al. 2013). In a recent study, Kopp et al. (2013) observed considerable amounts of 5FU on infusion pumps and considered high risk of exposure linked to the fact that this device was sometimes handled without gloves. The same type of results was published earlier by Fransman and colleagues in 2007. Additionally, a study developed by Hon and colleagues (2011) stated that these infusion pumps was the most contacted object by workers during drug administration.

Our results showed also that some surfaces $(8.6 \%)$ were contaminated with more than one antineoplastic drugs. Additionally, most of these samples were from the administration units. Our results can be explained once again by the fact that there are more strict safety and hygiene rules in preparation units when compared with administration units as demonstrated by the high number of organizations that research on this field developing constantly new rules and safety measures (ISOPP 2007; NIOSH among others).

This raises attention to another important aspect that should be considered for the risk assessment process: in this setting, exposure is characterized by being simultaneous to different antineoplastic drugs and the health effects of such mixtures are unpredictable (Fucic et al. 1998; Cavallo et al. 2005; Kopjar et al. 2009).

Although the discussion of data was based on the results obtained in the samples that revealed levels of contamination possible to quantify ( $>$ LOQ), we cannot forget that was also found a significant number of samples that was possible to detect one or more drug (35.8\%) (<LOQ) (Tables 5 and 6). This data is also relevant, considering that the risk associate to these drugs are more related with the exposure than with a dose, due to fact that the health effects related can be observe (even with lower probability) with only one contact or moment of exposure. Moreover, when performing statistical analysis of this data, administration units also presented the worst results, supporting the previous results and comments.

In view of the results obtained, some recommendations must be pointed out, namely the following: change the gloves more often, the trays must be used for only one admixture and then cleaned and the worktops and administration devices must be cleaned more frequently. 
As recommended by Hedmer et al. (2005a), we consider relevant to study in more detail how the levels of surface contamination varies over time in workplaces that handle antineoplastic drugs. The obtained data in this research only permits to consider these workplaces as contaminated environments and all the safety precautions must be applied.

\section{Conclusions}

Our results show that there is a real risk of exposure in the preparation and administration units of the studied hospitals due to the contamination found in many different and frequently touched surfaces.

The obtained results revealed that administration areas of both hospitals have higher levels of contaminated surfaces than preparation areas. Factors contributing to this are related with different work practices and different levels of awareness to the exposure problem. Additionally, the results point out to poor cleaning practices and/or incorrect working procedures.

Considering the findings, specific measures should be taken in both hospitals, namely the following: review the cleaning protocols (surfaces to clean, cleaning products and cleaning frequency); constantly promote good practices and safety procedures among all the professionals that handle antineoplastic drugs such as remove gloves immediately after drug handling and always before touching any surface; and implement routine monitoring of surfaces contamination in order to guarantee the appliance of safety measures and procedures.

Acknowledgments The study would not have been possible to develop without the financial support given by Portuguese Authority of Working Conditions (Project reference: 036APJ/09).

\section{References}

Acampora, A., Castiglia, L., Miraglia, N., Pieri, M., Soave, C., Liotti, F., et al. (2005). A case study: surface contamination of cyclophosphamide due to working practices and cleaning procedures in two Italian hospitals. Annals of Occupational Hygiene, 49(7), 611-618.

Brouwers, E. E. M., Huitema, A. D. R., Bakker, E. N., Douma, J. W., Schimmel, K. J. M., van Weringh, G., et al. (2007). Monitoring of platinum surface contamination in seven Dutch hospital pharmacies using inductively coupled plasma mass spectrometry. International Archives of Occupational and Environmental Health, 80(8), 689-699.

Castiglia, L., Miraglia, N., Pieri, M., Simonelli, A., Basilicata, P., Genovese, G., et al. (2008). Evaluation of occupational exposure to antiblastic drugs in an Italian hospital oncological department. Journal of Occupational Health, 50, 48-56.

Cavallo, D., Ursini, C. L., Perniconi, B., Francesco, A. D., Giglio, M., Rubino, F. M., et al. (2005). Evaluation of genotoxic effects induced by exposure to antineoplastic drugs in lymphocytes and exfoliated buccal cells of oncology nurses and pharmacy employees. Mutation Research, 587(1-2), 45-51.

Connor, T. H., \& McDiarmid, M. A. (2006). Preventing occupational exposures to antineoplastic drugs in health care settings. CA: A Cancer Journal for Clinicians, 56(6), 354-365.

Connor, T. H., Sessink, P. J., Harrison, B. R., Pretty, J. R., Peters, B. G., Alfaro, R. M., et al. (2005). Surface contamination of chemotherapy drug vials and evaluation of new vial-cleaning techniques: results of three studies. American Journal of Health-System Pharmacy, 62(5), 475-484.

Connor, T. H., DeBord, G., Pretty, J. R., Oliver, M. S., Roth, T. S. H., Lees, P. S., et al. (2010). Evaluation of antineoplastic drug exposure of health care workers at three university-based US cancer centers. JOEM, 52, 1019-1027.

Davis, J., McLauchlan, R., \& Connor, T. H. (2011). Exposure to hazardous drugs in healthcare: an issue that will not go away. Journal of Oncology Pharmacy Practice, 17(1), 9-13.

Delporte, J. P., Chenoix, P., \& Hubert, P. H. (1999). Chemical contamination of the primary packaging of 5-fluorouracil RTU solutions commercially available on the Belgian market. European Journal of Hospital Pharmacy, 5, 119-121.

Favier, B., Gilles, L., Ardiet, C., \& Latour, J. F. (2003). External contamination of vials containing cytotoxic agents supplied by pharmaceutical manufacturers. Journal of Oncology Pharmacy Practice, 9, 15-20.

Fransman, W., Vermeulen, R., \& Kromhout, H. (2004). Occupational dermal exposure to cyclophosphamide in Dutch hospitals: a pilot study. Annals of Occupational Hygiene, 48(3), 237-244.

Fransman, W., Vermeulen, R., \& Kromhout, H. (2005). Dermal exposure to cyclophosphamide in hospitals during preparation, nursing and cleaning activities. International Archives of Occupational and Environmental Health, 78(5), 403-412.

Fransman, W., Peelen, S., Hilhorst, S., Roeleveld, N., Heederik, D., \& Kromhout, H. (2007). A pooled analysis to study trends in exposure to antineoplastic drugs among nurses. Annals of Occupational Hygiene, 51(3), 231-239.

Fucic, A., Jazbec, A., Mijic, A., Seso-Simic, D., \& Tomek, R. (1998). Cytogenetic consequences after occupational exposure to antineoplastic drugs. Mutation Research, 416(1-2), 59-66.

Hedmer, M., Jönsson, B. A. G., \& Nygren, O. (2004). Development and validation of methods for environmental monitoring of cyclophosphamide in workplaces. Journal of Environmental Monitoring, 6, 979-984.

Hedmer, M., Georgiadi, A., Bremberg, E. R., Jönsson, B. A. G., \& Eksborg, S. (2005). Surface contamination of cyclophosphamide packaging and surface contamination with antineoplastic drugs in a hospital pharmacy in Sweden. Annals of Occupational Hygiene, 49(7), 629-637a.

Hedmer, M., Tinnerberg, H., Axmon, A., \& Jönsson, B. A. (2008). Environmental and biological monitoring of antineoplastic 
drugs in four workplaces in a Swedish hospital. International Archives of Occupational and Environmental Health, 81(7), 899-911.

Hirst, M., Tse, S., Mills, D. G., Levin, L., \& White, D. F. (1984). Occupational exposure to cyclophosphamide. 1(8370), 186188.

Hon, C. Y., Teschke, K., Chua, P., Venners, S., \& Nakashima, L. (2011). Occupational exposure to antineoplastic drugs: identification of job categories potentially exposed throughout the hospital medication system. Safety and Health at Work, 2(3), 273-281.

Hon, C. Y., Teschke, K., Chu, W., Demers, P., \& Venners, S. (2013). Antineoplastic drug contamination of surfaces throughout the hospital medication system in Canadian hospitals. Journal of Occupational and Environmental Hygiene, 10(7), 374-383.

Huang, Y. W., Jian, L., Zhang, M. B., Zhou, Q., Yan, X. F., Hua, X. D., et al. (2012). An investigation of oxidative DNA damage in pharmacy technicians exposed to antineoplastic drugs in two Chinese hospitals using the urinary $8-\mathrm{OHdG}$ assay. Biomedical and Environmental Sciences, 25(1), 109-116.

International Agency for Research on Cancer (IARC). (2012). Monographs on the evaluation of carcinogenic risks to humans. Some antiviral and antineoplastic drugs, and other pharmaceutical agents. Lyon, France: IARC Available from http://monographs.iarc.fr. Accessed 25 Oct 2013.

International Society of Oncology Pharmacy Practitioners ISOPP. (2007). ISOPP standards of practice: safe handling of cytotoxics. Journal of Oncology Pharmacy Practice, 13(Suppl), $1-81$.

Kiffmeyer, T. K., Tuerk, J., Hahn, M., Stuetzer, H., Hadtstein, C., Heinemann, A., et al. (2013). Application and assessment of a regular environmental monitoring of the antineoplastic drug contamination level in pharmacies - the MEWIP Project. Annals of Occupational Hygiene, 57(4), 444-455.

Kopjar, N., Garaj-Vrhovac, V., Kasuba, V., Rozgaj, R., Ramić, S., Pavlica, V., et al. (2009). Assessment of genotoxic risks in Croatian health care workers occupationally exposed to cytotoxic drugs: a multi-biomarker approach. International Journal of Hygiene and Environmental Health, 212(4), 414-431.

Kopp, B., Schierl, R., \& Nowak, D. (2013). Evaluation of working practices and surface contamination with antineoplastic drugs in outpatient oncology health care settings. International Archives of Occupational and Environmental Health, 86(1), 47-55.

Kromhout, H., Hoek, F., Uitterhoeve, R., Huijbers, R., Overmars, R. F., Anzion, R., et al. (2000). Postulating a dermal pathway for exposure to anti-neoplastic drugs among hospital workers. Applying a conceptual model to the results of three workplace surveys. Annals of Occupational Hygiene, 44(7), 551-560.

Larson, R. R., Khazaeli, M. B., \& Dillon, H. K. (2002). Monitoring method for surface contamination caused by selected antineoplastic agents. American Journal of HealthSystem Pharmacy, 59(3), 270-277.

Larson, R. R., Khazaeli, M. B., \& Dillon, H. K. (2003). A new monitoring method using solid sorbent media for evaluation of airborne cyclophosphamide and other antineoplastic agents. Applied Occupational and Environmental Hygiene, 18(2), 120-131.
Mason, H. J., Morton, J., Garfitt, S. J., Iqbal, S., \& Jones, K. (2003). Cytotoxic drug contamination on the outside of vials delivered to a hospital pharmacy. Annals of Occupational Hygiene, 47(8), 681-685.

Mason, H. J., Blair, S., Sams, C., Jones, K., Garfitt, S. J., Cuschieri, M. J., et al. (2005). Exposure to antineoplastic drugs in two UK hospital pharmacy units. Annals of Occupational Hygiene, 49(7), 603-610.

McDevitt, J. J., Lees, P. S., \& McDiarmid, M. A. (1993). Exposure of hospital pharmacists and nurses to antineoplastic agents. Journal of Occupational Medicine, 35(1), 57-60.

Minoia, C., Turci, R., Sottani, C., Schiavi, A., Perbellini, L., Angeleri, S., et al. (1998). Application of high performance liquid chromatography/tandem mass spectrometry in the environmental and biological monitoring of health care personnel occupationally exposed to cyclophosphamide and ifosfamide. Rapid Communications in Mass Spectrometry, 12(20), 1485-1493.

Miyake, T., Iwamoto, T., Tanimura, M., \& Okuda, M. (2013). Impact of closed-system drug transfer device on exposure of environment and healthcare provider to cyclophosphamide in Japanese hospital. SpringerPlus; 2: 273. Available from http://www.springerplus.com/content/2/1/273. Accessed 25 Oct 2010.

Nygren, O., Gustavsson, B., Ström, L., \& Friberg, A. (2002). Cisplatin contamination observed on the outside of drug vials. Annals of Occupational Hygiene, 46(6), 555-557.

Schierl, R., Böhlandt, A., \& Nowak, D. (2009). Guidance values for surface monitoring of antineoplastic drugs in German pharmacies. Annals of Occupational Hygiene, 53(7), 703-711.

Schmaus, G., Schierl, R., \& Funck, S. (2002). Monitoring surface contamination by antineoplastic drugs using gas chromatography-mass spectrometry and voltammetry. American Journal of Health-System Pharmacy, 59(10), 956-961.

Sessink, P. J., Boer, K. A., Scheefhals, A. P., Anzion, R. B., \& Bos, R. P. (1992). Occupational exposure to antineoplastic agents at several departments in a hospital. Environmental contamination and excretion of cyclophosphamide and ifosfamide in urine of exposed workers. International Archives of Occupational and Environmental Health, 64(2), 105-112.

Sessink, P. J., Van de Kerkhof, M. C., Anzion, R. B., Noordhoek, J., \& Bos, R. P. (1994). Environmental contamination and assessment of exposure to antineoplastic agents by determination of cyclophosphamide in urine of exposed pharmacy technicians: is skin absorption an important exposure route? Archives of Environmental Health, 49(3), 165-169.

Shirato, S. (1992). Problems of cytotoxicity drug mainly based on antineoplastic tumor medicine. Japanese Journal of Medical Waste Research, 5, 1-32.

Sugiura, S., Asano, M., Kinoshita, K., Tanimura, M., \& Nabeshima, T. (2011). Risks to health professionals from hazardous drugs in Japan: a pilot study of environmental and biological monitoring of occupational exposure to cyclophosphamide. Journal of Oncology Pharmacy Practice, 17(1), 14-19.

Touzin, K., Bussières, J. F., Langlois, E., \& Lefebvre, M. (2009). Evaluation of surface contamination in a hospital hematology-oncology pharmacy. Journal of Oncology Pharmacy Practice, 15, 53-61. 
Touzin, K., Bussières, J. F., Langlois, E., Lefebvre, M., \& Métra, A. (2010). Pilot study comparing the efficacy of two cleaning techniques in reducing environmental contamination with cyclophosphamide. Annals of Occupational Hygiene, 54(3), 351-359.

Turci, R., Sottani, C., Spagnoli, G., \& Minoia, C. (2003). Biological and environmental monitoring of hospital personnel exposed to antineoplastic agents: a review of analytical methods. Journal of Chromatography, B: Analytical Technologies in the Biomedical and Life Sciences, 789(2), 169-209.

Turci, R., Minoia, C., Sottani, C., Coghi, R., Severi, P., Castriotta, C., et al. (2011). Occupational exposure to antineoplastic drugs in seven Italian hospitals: the effect of quality assurance and adherence to guidelines. Journal of Oncology Pharmacy Practice, 17(4), 320-332. doi:10.1177/ 1078155210381931

Yang, T., Cui, F., Choi, M., Lin, H., Chung, S., Shim, C., \& Kim, D. (2007). Liposome formulation of paclitaxel with enhanced solubility and stability. Drug Delivery, 14(5), 301-308.

Yoshida, J., Koda, S., Nishida, S., Nakano, H., Tei, G., \& Kumagai, S. (2013). Association between occupational exposure and control measures for antineoplastic drugs in a pharmacy of a hospital. Annals of Occupational Hygiene, 57(2), 251-260. 